\title{
Barriers, Enablers, and Incentives for Research Participation: A Report from the Ambulatory Care Research Network (ACRN)
}

\author{
Suzanne Bakken, RN, DNSc, Rafael A. Lantigua, MD, Linda V. Busacca, BA, and \\ J. Thomas Bigger, $M D$
}

Background: Supported by a supplement to our Clinical and Translational Science Award, we studied the feasibility of implementing clinical research in Northern Manhattan community practices that primarily serve Hispanic patients.

Methods: We applied a mixed-methods approach (surveys, focus groups, interviews) based on the PRECEDE-PROCEED model to determine the level of interest in clinical research among community clinicians (both practice-based research network [PBRN] members and non-PBRN members), the perceived barriers that hamper participation in clinical research, and the perceived facilitators for conducting research in such practices.

Results: Survey and qualitative data indicated strong interest in clinical research among current and potential PBRN members if it was relevant to improving quality of care in their practice or community. They also identified important perceived barriers (lack of time, inadequate training in research methods, lack of collaborators and support staff, institutional review board hurdles, and community distrust of research) and the necessary requirements for overcoming barriers to conducting research in busy clinical settings, which included collaborators, mentors, research support staff, and a trusting patientclinician relationship.

Conclusion: It is feasible to conduct clinical research studies in urban community medical practices if the topics are relevant to the community and appropriate enabling structures and processes are put into place. (J Am Board Fam Med 2009;22:436-45.)

The National Institutes of Health (NIH) Roadmap program has developed initiatives related to building clinical research capability in community medical practices so that patients can participate in such studies at their primary point of contact with the

This article was externally peer reviewed.

Submitted 29 January 2009; revised 26 April 2009; accepted 29 April 2009.

From the School of Nursing and Department of Biomedical Informatics (SB); the Department of Medicine, Division of General Internal Medicine (RAL); the Clinical Trials Network, Clinical Trials Office (LVB), and the Departments of Medicine and of Pharmacology (JTB), Columbia University, New York, NY.

Funding: This project was funded by grants and contracts from National Institutes of Health: 5UL1RR024156 and 3UL1RR024156-02S1, contract no. HHSN2682004 55208C.

Conflict of interest: none declared.

Corresponding author: Suzanne Bakken, RN, DNSc, FAAN, School of Nursing and Department of Biomedical Informatics, $630 \mathrm{~W} .168^{\text {th }}$ Street, Columbia University, New York, NY (E-mail: sbh22@columbia.edu). health care system. ${ }^{1-5}$ Roadmap initiatives are designed to improve the access of patients to NIHtype clinical research, increase the racial and ethnic diversity of participants, and enhance the translation of research into practice. ${ }^{6}$ The last objective, in particular, is consistent with the role played by practice-based research networks (PBRNs). ${ }^{7-9}$ In addition, a 2007 survey of 89 PBRNs reported that patients of minority race, Hispanic ethnicity, and low socioeconomic status were overrepresented in PBRNs as compared with the general population and those seeking health care. ${ }^{8}$ In a recent commentary regarding transformation of health care at the front line, Conway and Clancy ${ }^{10}$ note that the "testing, scaling, and spreading of interventions to improve quality and efficiency needs to be performed via learning networks led by clinicians and other stakeholders." These factors suggest that PBRNs play an important role in achieving Roadmap objectives. 
Although some concern has been expressed regarding a perceived shift in strategic direction at the federal level from "direct funding of a harmonized national infrastructure of cooperating networks to a model of local engagement of primary care clinics performing practice-based research under the aegis of regional academic health centers through Clinical and Translational Science Awards [CTSA]" (p. 229) ${ }^{11}$ our perspective is that the CTSA approach and the historical role of PBRNs can be complementary efforts. Consequently, through a supplement to our CTSA, we studied the feasibility of implementing clinical research in urban community practices in Northern Manhattan. ${ }^{6}$ We applied a mixed-methods approach informed by the predisposing, reinforcing, and enabling constructs of the PRECEDE-PROCEED model ${ }^{12}$ to collect data from clinicians in community practices to determine the level of interest in clinical research, the perceived barriers that hamper the participation of community medical practices in clinical research, and the perceived facilitators for conducting research in such practices.

This report primarily focuses on physicians in Ambulatory Care Network (ACN) sites in Northern Manhattan, New York City. The ACN was registered as a PBRN, the Ambulatory Care Research Network, in the Agency for Health Care Research and Quality database in 2006. In addition, where relevant we contrast the ACN data with that of clinicians from community practices with the potential to serve as research sites. We also discuss possible solutions to key perceived barriers in the $\mathrm{ACN}$ and potential research sites, drawing on the PBRN literature and our experience in the conduct of multisite clinical trials.

\section{Methods \\ Setting}

The ACN is a large network of primary care and specialty clinics affiliated with New York Presbyterian Hospital, which serves the greater New York area. In Northern Manhattan (Washington Heights, Inwood, and Harlem) there are 8 ACN community primary care practice sites, 6 of which have internal medicine or family medicine clinicians. ACN physicians are faculty members at the College of Physicians and Surgeons, Columbia University, and their role expectations include resident training and clinical scholarship in addition to clinical responsibilities. Annual ACN outpatient visits total approximately 750,000; approximately one-third of these occur at Northern Manhattan clinics. The majority of primary care patients are covered by Medicaid programs (25\% fee-for-service Medicaid and 40\% Medicaid Managed Care). The main area served by the Northern Manhattan ACN clinics (Washington Heights/Inwood) is primarily Hispanic (74.1\%).

\section{Sample}

The primary sample for this report was ACN physicians. Two additional groups of clinicians from potential community research sites participated in either a focus group or interview: Hispanic physicians not affiliated with the ACN and doctorally prepared nurse practitioners (DNPs) from the faculty practice of the Columbia University School of Nursing, Columbia Advanced Practice Nurse Associates. These clinicians had research qualifications similar to the ACN physicians and were recruited for an earlier phase of our research. They are included as a contrast to ACN physicians.

\section{Institutional Review Board}

The study was approved by Columbia University Medical Center Institutional Review Board. Participants provided verbal consent for participation after reviewing an information sheet and were compensated for focus group or interview participation but not for completing the survey.

\section{Recruitment}

Recruitment methods varied depending on the method judged to be most appropriate for the target group of participants. ACN physicians, the primary sample for this report, were recruited through an e-mail invitation to complete the survey. Those that completed the survey were subsequently invited to participate in a focus group in 1 of $3 \mathrm{ACN}$ sites. A purposive sample of 5 community-based Hispanic physicians was nominated by a member of the research team (RAL) and personally invited to participate in an individual interview. DNPs were recruited through an e-mail invitation for focus group or interview participation.

\section{Study Instruments}

We used the predisposing, reinforcing, and enabling constructs of the PRECEDE-PROCEED model $^{12}$ as the theoretical basis for developing 
quantitative and qualitative questions related to clinical research participation. Although the model is more typically applied to health behaviors, we considered clinical research participation to be a clinician behavior and felt that our understanding of barriers and facilitators could be informed by the model. Predisposing factors precede a behavior and can be thought of as motivation or "wanting to do." ${ }^{13}$ For our study, this was characterized as clinician interest. Enabling factors are those related to "being able to do," and for this study were the skills and resources required to conduct clinical research. Reinforcing factors are "rewards for doing" and included positive (eg, monetary or academic incentives) and negative (eg, concerns about clinical productivity) feedback received as a result of clinical research participation. Conceptualizing barriers and facilitators for clinical research in this way provides guidance for a hierarchy of potential interventions or solutions starting from strengthening interest through enabling and rewarding clinical research participation.

Based on the model, existing surveys, and our prior experience, we constructed a 40-item survey that collected information about provider demographics, clinical and research training, previous research experience, their current clinical practice and research activity, level of interest in future clinical research, barriers and incentives to participation in clinical research, and the kinds of clinical research the providers most want to conduct. Questions related to interest, barriers, and incentives were rated on a 5 -point Likert scale, from strongly agree to strongly disagree (the questionnaire is available by request to the corresponding author.)

Focus group and interview questions were openended and targeted perceptions of barriers and facilitators related to conducting research in their setting. Questions were preceded by a general introduction to the CTSA initiative and the specific study purpose. Although general questions remained consistent during the data collection, probes were updated to reflect themes identified as the data collection and analysis evolved.

\section{Data Collection}

ACN physicians completed the survey online using SurveyMonkey (SurveyMonkey, Portland, OR) before participation in a focus group. We conducted 3 focus groups with internal medicine and family medicine physicians in the ACN. Before the data collection in the ACN, we also conducted 1 focus group and 6 individual interviews with clinicians from potential community research sites in an initial phase of the research. All focus groups and interviews were led by the same investigator (SB) and lasted approximately 1 to 1.5 hours.

\section{Data Analysis}

Survey responses were summarized using descriptive statistics. Data analysis of the qualitative data began with peer debriefing among research team members at the conclusion of the interviews or focus groups. Subsequently, transcripts of the focus groups were iteratively coded and analyzed by one investigator (SB) to generate major themes and categories. Other research team members reviewed themes and categories and the assignment of utterances to themes and categories. Suggestions for revisions were discussed among team members and final decisions were based on consensus. For the ACN physicians, qualitative data were compared with survey data to determine instances in which the qualitative data expanded in survey responses and instances in which the qualitative data contributed information not identified through the survey. Because the focus of this article is on PBRNs, qualitative data from clinicians in potential community research sites are only included in the reported results when they illustrate a new theme that was not identified from the ACN data or strongly confirm ACN data.

\section{Results}

\section{Participants}

Fifty-four surveys were distributed. Fourteen internal medicine and 10 family medicine ACN physicians completed the survey; response rate was $44 \%$. The average age of the participants was 43 years and $76 \%$ were women. Self-reported race was $48 \%$ white, 16\% Asian, 12\% black, and 24\% selected other or declined to state. Twenty-four percent were of Hispanic ethnicity. Three-fourths were married or partnered, and half had children. Twenty-five percent of the ACN physicians were foreign medical graduates. Practice activity is summarized in Table 1.

Twenty-five percent of the ACN physicians had formal research training and $75 \%$ had some research experience (Table 2). Only $21 \%$ had clinical 
Table 1. Practice Activity of the Ambulatory Care Network Physicians $(\mathrm{n}=\mathbf{2 4})$

\begin{tabular}{lcr}
\hline & Mean \pm SD & Range \\
\hline Years in practice (n) & $11 \pm 8$ & $1-28$ \\
Commute time (minutes) & $36 \pm 16$ & $7-60$ \\
Practice hours per work week (n) & $43 \pm 14$ & $9-70$ \\
Time with patients (\%) & $48 \pm 24$ & $13-80$ \\
Patients seen per day (n) & $13 \pm 5$ & $5-20$ \\
$\quad$ African-American patients (\%) & $13 \pm 10$ & $0-40$ \\
$\quad$ Hispanic patients (\%) & $77 \pm 20$ & $15-95$ \\
\hline
\end{tabular}

research training certification. Most of the clinicians $(81 \%)$ with previous research experience found it a positive experience. Thirty-eight percent

Table 2. Research Training and Experience of the Ambulatory Care Network Physicians $(n=24)$

\begin{tabular}{|c|c|}
\hline Formal research training & Percent \\
\hline None & 76 \\
\hline Research fellowship & 16 \\
\hline Research-oriented master's degree & 16 \\
\hline Research-oriented doctoral degree & 0 \\
\hline \multicolumn{2}{|l|}{ Research experience } \\
\hline None & 24 \\
\hline Retrospective studies (chart reviews) & 28 \\
\hline Prospective observational studies & 24 \\
\hline Behavioral intervention & 16 \\
\hline Health services/outcomes research & 40 \\
\hline Clinical trials & 24 \\
\hline \multicolumn{2}{|l|}{ Research sponsors (funding) } \\
\hline Industry & 4 \\
\hline Foundations & 8 \\
\hline City or state & 12 \\
\hline Agency for Healthcare Research and Quality & 4 \\
\hline Centers for Disease Control and Prevention & 4 \\
\hline National Institutes of Health & 12 \\
\hline $\begin{array}{l}\text { Other (mostly various medical school funds for } \\
\text { students) }\end{array}$ & 44 \\
\hline \multicolumn{2}{|l|}{ Research training certificates } \\
\hline None & 76 \\
\hline Good clinical practices & 20 \\
\hline National Institutes of Health & 0 \\
\hline Association of Clinical Research Professionals & 0 \\
\hline Other (medical school elective) & 4 \\
\hline \multicolumn{2}{|l|}{ Satisfaction with previous research experiences } \\
\hline Positive & 81 \\
\hline Neutral & 13 \\
\hline Negative & 6 \\
\hline $\begin{array}{l}\text { Currently involved in any research projects } \\
\text { (NIH, Health Services) }\end{array}$ & 36 \\
\hline Currently employs a clinical research coordinator & 12 \\
\hline
\end{tabular}

NIH, National Institutes of Health. were currently involved in a clinical research project primarily sponsored by the NIH or health services agencies.

Focus group and interview participants included 13 ACN physicians (8 internal medicine and 5 family medicine); 5 community-based Hispanic physicians ( 4 men and 1 women, each of whom was board-certified in one or more specialties); and all DNPs $(n=4)$ in the faculty practice (all of whom were women and board-certified in their specialty).

\section{Clinician Interest in Research}

Survey, focus group, and interview data strongly support clinician interest in research, an essential predisposing factor for research participation. A key motivation was the relevance of the research topic to quality of care in their setting. Top-ranked types of clinical research in terms of ACN physician interest were health services/outcomes research (88\%), behavioral intervention (73\%), observational (71\%), retrospective (58\%), dissemination (48\%), and clinical trials (26\%). All focus group and interview participants expressed some level of interest in research, ranging from research facilitation to serving as a site investigator for multisite clinical trials. Eighty percent of the ACN survey respondents indicated a willingness to deliver an intervention per research protocol. All DNPs had completed research course requirements as part of their clinical doctorate program. ${ }^{14}$

\section{Barriers and Facilitators (Enabling and Reinforcing Factors)}

Tables 3 and 4 display ACN study results related to barriers and facilitators (enabling and reinforcing factors) in 3 parts: (1) items found in both the survey and focus groups, (2) survey responses only, and (3) additional thematic categories from the focus group data. In terms of perceived barriers (Table 3), clinician time and training appeared in both survey and focus group data, whereas support staff-related barriers (eg, time, compensation, training) were identified only in the survey. Lack of time/competing demands for time was the topranked barrier $(92 \%)$ in the survey, and $80 \%$ of ACN clinicians agreed that their lack of appropriate training was a barrier. Four thematic categories emerged only in the focus group data: (1) difficulty filling clinical sessions to enable release for compensated research time, (2) lack of collaborators to sustain research, (3) difficulties overcoming institu- 
Table 3a. Barriers to Ambulatory Care Network Physician Participation in Research Identified in Both Survey and Focus Groups

\begin{tabular}{|c|c|c|}
\hline \multicolumn{2}{|l|}{ Survey } & \multirow[b]{2}{*}{ Participant's Statements from Focus Group } \\
\hline Item & Physicians Who Agree (\%)* & \\
\hline Lack of time/competing demands for time & 92.0 & $\begin{array}{l}\text { - Am I interested in research? I am really interested } \\
\text { in research. Am I interested in being reimbursed } \\
\text { for research? I am, but right now I have only } 5 \\
\text { clinical sessions a week and one of those I precept } \\
\text { the residents. So I only see patients } 4 \text { sessions a } \\
\text { week. I don't think I can go down to } 3 \text { and still call } \\
\text { myself a primary care doctor so easily, or I don't } \\
\text { know if I am ready to go down to 3. And so, again, } \\
\text { we wear so many hats and, yes, I would love to } \\
\text { wear another hat. (IM) } \\
\text { - The largest barrier is basically time. There is no } \\
\text { time to do any of this, or it's going to be in your } \\
\text { sleep. ...it's been going on for } 3 \text { years, and it's } \\
\text { difficult. (FM) } \\
\text { - Coming from residency, if you haven't gone into a } \\
\text { program that fosters research you don't have the } \\
\text { skills in order to write grants or be the principal } \\
\text { investigator of a research project, ...unless you've } \\
\text { had a mentor who's actually taught you how to do } \\
\text { that, so adequate skills or training.... (IM) }\end{array}$ \\
\hline My lack of appropriate training & 80.0 & $\begin{array}{l}\text { - We don't really understand what it entails. In terms } \\
\text { of what do we have to put in to it really ... and how } \\
\text { much do we want to put in? Do we want to be the } \\
\text { PIs, or do we want to be involved in projects with } \\
\text { other people that are staying up until 3:00 in the } \\
\text { morning so we don't have to? (FM) } \\
\text { - And not getting reimbursed for it makes a huge } \\
\text { difference (IM) }\end{array}$ \\
\hline Inadequate compensation for my time & 79.2 & $\begin{array}{l}\text { - It's [the grant] been going on for } 3 \text { years, and it's } \\
\text { difficult. My time is not bought out; it's in kind. So } \\
\text { this is on top of all of the things that I'm doing. } \\
\text { (FM) }\end{array}$ \\
\hline
\end{tabular}

*Percent of participants selecting strongly agree or agree in response to statement; only statements that $>50 \%$ participants rated as strongly agree or agree are included.

PI, primary investigator; IM, internal medicine; FM, family medicine.

tional review board hurdles, (4) and community distrust of research.

Potential researchers from community sites described several barriers that did not surface in the ACN focus groups. The comments of Hispanic physician respondents reflected the financial responsibility associated with their independent prac-

Table 3b. Barriers Identified in Survey Only

\begin{tabular}{lc}
\hline Item & $\begin{array}{c}\text { Physicians Who } \\
\text { Agree }(\%)^{*}\end{array}$ \\
\hline Support staff time & 66.7 \\
Inadequate compensation for support & 62.5 \\
$\quad$ staff time & 58.4 \\
Support staff training & \\
\hline
\end{tabular}

*Percent of participants selecting strongly agree or agree in response to statement; only statements that $>50 \%$ participants rated as strongly agree or agree are included. tices and highlighted the need to have the research studies fit into clinical workflow and not negatively affect the bottom line. For instance, one physician said, "Anything that makes me move in one direction, more than one iota....can decrease the efficiency to the point that actually patient care starts to suffer...there's no fat in private practice-we basically deal with bare bones." Hispanic physicians in independent community practices also described additional sociocultural issues, including language, literacy, complexity of patients' lives, and the research protocol challenges related to community members who travel between New York and the Dominican Republic.

ACN clinicians described the importance of enabling factors such as collaborators, mentors, and research support staff to overcome the barriers to conducting research in busy clinical settings (Table 


\begin{tabular}{|c|c|}
\hline Themes & Participant's Statements \\
\hline $\begin{array}{l}\text { Difficulty filling clinical sessions to enable } \\
\text { release for compensated research time }\end{array}$ & $\begin{array}{l}\text {-You have to consider that when a peg is gone, there's a hole there, } \\
\text { and how do you fill that hole? And then will it get to a critical } \\
\text { point where there is no more leftover pegs to fill in. When you } \\
\text { pull someone to cover that, you're pulling them from something } \\
\text { else. There is a domino effect. You can't hire, appoint one person } \\
\text { to cover that hole, either, even if you have the release money. } \\
\text { And then what happens when she loses her funding? Do you } \\
\text { throw away that person we hired? Then it becomes a budget } \\
\text { issue. (FM) }\end{array}$ \\
\hline Lack of collaborators to sustain research & $\begin{array}{l}\text { - And then we did the survey and having to get it translated, right, } \\
\text { then we realized the survey was too complicated for our patients } \\
\text { so we needed then to simplify and then we had to resubmit it. I } \\
\text { mean, it was just exhausting. So we did it and we did quite a few } \\
\text { of them, but we never did anything with it because again you } \\
\text { need a group of people who can consistently meet. (IM) } \\
\text { It's just very hard to sustain things. That sustainability is one of } \\
\text { my biggest challenges. (IM) }\end{array}$ \\
\hline Difficulties overcoming IRB hurdles & $\begin{array}{l}\text { - I can't believe how hard it was to get IRB approval for something } \\
\text { that involved no drugs. You know, this couldn't have been more } \\
\text { soft research. And it's just because it doesn't matter what it is, } \\
\text { you have to go through all the steps no matter what. And it was } \\
\text { so hard and so many back and forths in getting the consents } \\
\text { approved and being confused about whether...oh, I was originally } \\
\text { supposed to include kids so I needed assent and consent and kids } \\
\text { never came, thank goodness. . . and the grant was written for } \\
\text { like } 100 \text { people and I got } 20 \text { at the beginning and then } 10 \text { and } \\
\text { then } 6 \text {. (IM) } \\
\text { I have projects and I tried to get IRB approval. And I finally } \\
\text { found someone I thought could help me on the process. They did } \\
\text { help me, but when I submitted it, it still got denied, and then we } \\
\text { couldn't figure out how to overcome that barrier. So we get to a } \\
\text { certain point. We marshal all of our time and energy and } \\
\text { resources, and we get to a certain point and reach another hurdle, } \\
\text { and we just drop it. (FM) }\end{array}$ \\
\hline Community distrust of research & $\begin{array}{l}\text { - . . Spanish was my primary language and I feel like I have a } \\
\text { special connection with them because I speak exactly as they do, } \\
\text {. . . they feel that you are using them sometimes. They don't trust } \\
\text { you enough. (IM) } \\
\text {. . . I can speak from the African American population that there's } \\
\text { a lot of mistrust of the medical community. . . They don't want } \\
\text { to be experimented on, especially after that whole Tuskegee } \\
\text { experiment. (IM) }\end{array}$ \\
\hline
\end{tabular}

IRB, institutional review board; FM, family medicine; IM, internal medicine.

4). The data from the Hispanic physicians was consistent with that from the ACN physicians in the need for funded research support staff, but also mentioned the importance of a respectful relationship between research partners from academia and those in the community. For example, one Hispanic physician noted, "I guess one of the most important things when you want to do this-especially with people who never had any experience[is] a good rapport...treat others with respect; it's something very important."

The ACN physicians, Hispanic physicians, and DNPs all described the important role of the patientprovider relationship in overcoming patients' reluctance for research participation. For example, one
Hispanic physician commented that “...the Hispanic patient will do most of the time whatever you ask them to do because they still they have the physician in very high regard-this guy is not going to do anything to harm me..." A DNP noted that "...if there is interest in the practitioners ... patients might follow through." During focus group discussions, ACN physicians also identified several specific strategies for engaging patients/community in research (Table 4).

Clinicians from potential research sites, including the DNP practice, described the important role of information systems, particularly electronic health records, in facilitating research primarily to search for potential research and patients and to retrieve data related to research variables. 
Table 4a. Facilitators for Ambulatory Care Network Physician Participation in Research Identified in Both Survey and Focus Group

\begin{tabular}{|c|c|c|}
\hline \multicolumn{2}{|l|}{ Survey } & \multirow[b]{2}{*}{ Participant's Statements from Focus Group } \\
\hline Item & Physicians Who Agree (\%)* & \\
\hline $\begin{array}{l}\text { Potential to improve care in my practice and } \\
\text { Contribution to knowledge development on } \\
\text { topic }\end{array}$ & 100 & $\begin{array}{l}\text { - And so any study that helps contribute to } \\
\text { our knowledge of what can help our patients } \\
\text { and what is the best practice is something } \\
\text { that this group is going to be interested in. } \\
\text { (IM) } \\
\text { - I have found people very open to working } \\
\text { with things if they're brought in a way that } \\
\text { their collaborating, inclusive, right, you } \\
\text { don't have to do this but we think this } \\
\text { might work, in a place that people can feel } \\
\text { like it's incorporating things into their } \\
\text { practice and not adding more work into } \\
\text { their practice. Key. (IM) }\end{array}$ \\
\hline Opportunity for my professional development & 100 & $\begin{array}{l}\text { - I think we are all academics and that's why } \\
\text { we're here. We're not in private practice for } \\
\text { a reason. (IM) }\end{array}$ \\
\hline Research collaborators/mentors & 92 & $\begin{array}{l}\text { - One of the issues with collaborators if we're } \\
\text { all primarily clinicians then it gets very } \\
\text { difficult because as clinicians we tend to put } \\
\text { the clinical first. The only reason why I } \\
\text { actually ended up finishing was because the } \\
\text { person I worked with was primarily a } \\
\text { researcher and he pushed and pushed } \\
\text { because he wanted to get this done so he } \\
\text { gave me deadlines. . (IM) } \\
\text { - That's the importance of mentorship, to } \\
\text { keep you from wasting your time on things } \\
\text { that you should not have to be focusing on. } \\
\text { (FM) }\end{array}$ \\
\hline
\end{tabular}

*Percent of participants selecting strongly agree or agree in response to statement; only statements that $>50 \%$ participants rated as strongly agree or agree are included.

IM, internal medicine; FM, family medicine.

Although respondents from all 3 groups $(\mathrm{ACN}$, independent Hispanic physician practices, and DNPs) addressed the importance of compensation for time spent on research-related tasks. A unique category that emerged from the Hispanic physician data was the need for a stable revenue stream from research: "Neither do we want our funding to be episodic."

Table 4b. Facilitators Identified in Survey Only

\begin{tabular}{lc}
\hline Item & $\begin{array}{c}\text { Physicians Who } \\
\text { Agree }(\%)^{*}\end{array}$ \\
\hline $\begin{array}{l}\text { Recognition as a member of a practice-based } \\
\text { research network }\end{array}$ & 66.6 \\
Recognition as a research collaborator & 64.0 \\
Continuing medical education credit & 60.0 \\
\hline
\end{tabular}

*Percent of participants selecting strongly agree or agree in response to statement; only statements that $>50 \%$ participants rated as strongly agree or agree are included.
In terms of reinforcing factors, all ACN clinicians identified the possibility of improving the quality of care in their practice site and contributing to knowledge development as incentives for research participation (Table 4). Other important incentives were professional development, continuing medical education, collaboration with academic researchers, and recognition as a PBRN member or research collaborator. Both ACN survey respondents $(60 \%)$ and Hispanic physicians in community sites identified authorship as an incentive, with one Hispanic physician stating, "...sooner or later, everybody [physicians] wants to see their name [in print].”

\section{Discussion}

\section{Clinician Interest in Research}

The survey and qualitative data provide evidence for the presence of clinician interest in participating in the current and future PBRN sites. They also highlight great interest and motivation (predispos- 


\begin{tabular}{|c|c|}
\hline Thematic Categories & Participant's Statements \\
\hline Research support staff & $\begin{array}{l}\text { - You need support of other staff that's going to help you to be } \\
\text { able to implement this research project. (IM) } \\
\text { - A definite support staff under research, not necessarily the top } \\
\text { people, but the bottom people, ... who can get data for you so } \\
\text { you don't have to spend time doing that. (FM) }\end{array}$ \\
\hline Patient/community engagement strategies & $\begin{array}{l}\text { - It's a matter of education in our community to try to convey the } \\
\text { information to our people that research is needed to be able to } \\
\text { understand how our community responds to treatments, the } \\
\text { management of disease, preventive medicine, and so forth; and } \\
\text { not just to rely on what other communities have done. . (IM) } \\
\text { - And having a relationship with your physician is a key part. I } \\
\text { mean, my patients, some of them I have been with for } 10 \text { years, I } \\
\text { see their family and I know this and that. . . there's a rapport } \\
\text { absolutely that would allow some of it to happen. (IM) } \\
\text { - And if it's just a survey and you can have them do it in your } \\
\text { waiting room, then you catch them and it's a little bit easier, } \\
\text { especially if you're handing them, whatever, a } \$ 10 \text { metro card or } \\
\$ 20 \text { gift card. (IM) }\end{array}$ \\
\hline
\end{tabular}

IM, internal medicine; FM, family medicine.

ing factors) for studies that are closely linked to improving the quality of care in the practice or that target important health problems in the community. This is consistent with the American Academy of Family Physicians National Research Network (AAFP NRN) recommendation to select studies that interest PBRN members and have the potential to improve quality of care. ${ }^{7}$ The high level of interest may reflect the fact that clinicians with some interest in clinical research were more likely to participate in our survey and focus groups or interviews and that research was a faculty role expectation of the ACN physicians. Approximately one-third of our clinician sample was Hispanic, and the level of interest contrasts with the findings of a national survey of randomly selected physicians that found that Hispanic physicians were less likely than non-Hispanic white physicians to be involved in clinical trials, enroll their patients in a treatment trial, and consider clinical trials to be of value. ${ }^{15}$ This difference is probably a result of our purposive rather than random sampling method.

\section{Enabling Factors}

Most of what we learned in our study related to skills and resources (enabling factors) for conducting clinical research because the participants were already predisposed to research participation. Many clinicians felt that they lacked adequate research skills and identified the need for research training in general and for particular tasks. Most $\mathrm{ACN}$ clinicians were unaware of local institutional resources, such as the CTSA-supported research training and design and biostatistics consultation services, that could meet these learning needs for those clinicians who want to lead studies. Settings that lack such core resources may access external educational resources. ${ }^{16-19}$

Clinicians identified a number of other potential enablers to decrease barriers and improve their ability to participate in research, including compensation for time used for research, academic collaborators, research support staff, mentorship, and electronic health records. Several factors primarily relate to one of the key strategies from AAFP NRN: creating a practical budget that covers the cost of the study. ${ }^{7}$ Part of the selection of projects for a PBRN is to make reasonably accurate estimates of the staff effort that will be needed for the project based on the protocol and to secure a budget that will adequately support the staff. When the geographic dispersion of practices and/or clinics permits, some of the personnel can work at more than one site, permitting the sites to share the financial burden of personnel. To make such arrangements work, AAFP NRN recommends securing written agreements among team members and provides policies and model agreements. ${ }^{20}$

Our experience with multisite clinical trials indicates that, if calculated correctly, fee-for-service reimbursement is fair and the revenue is automatically adjusted for level of effort. Two relatively common problems can reduce the potential benefits of this method: (1) the underestimation of the 
time to conduct the protocol and (2) inefficiency in conducting the protocol; thus, it is vital to include practices and front-line staff early in protocol development. Central support units that negotiate large numbers of budgets are likely to correctly estimate research-related effort. Inefficient sites can expend more time than is reimbursed even when reimbursement is correctly estimated. The latter problem can be addressed by additional training, assessment, and consultations that improve workflow efficiency. Improving enrollment usually improves efficiency because greater research activity facilitates the learning and retaining of knowledge of the protocol. Documenting research services so that reimbursement is correct and prompt can be facilitated by software that integrates workflow support and documents research services. ${ }^{21}$

Electronic health records were primarily mentioned as an enabling factor by clinicians not currently affiliated with the ACN to support tasks such as identification of potential research subjects and conduct health services research or epidemiologic studies. This has been successful in several PBRNs, ${ }^{22,23}$ and a number of institutions have made this a strategy in their CTSA activities.

The willingness of community members to participate in clinical research was of major interest to our research team for several reasons, including the historical lack of diversity in clinical research ${ }^{24}$ and the NIH Roadmap objective of increasing diversity in clinical research. Patient-related issues such as language, functional literacy, community distrust of research in general, and complex lives were identified by the clinicians. However, many clinicians, including the 5 Hispanic clinicians from non-ACN community practices, felt that if the research was highly relevant to the community (eg, hypertension, diabetes, asthma, or depression) and was supported by study materials appropriate in language and literacy, that the trusted relationship between clinician and patient would outweigh barriers to participation for many patients. This is consistent with the findings of 2 recent studies. ${ }^{25,26} \mathrm{Within}$ this trusted relationship, the tenets of informed consent must be carefully followed to avoid coercion.

Our institution has undertaken an additional strategy to enable patient participation in research: the establishment of a community health and information center outside of the medical center complex. The roles of this center, which is under the auspices of our CTSA, include informing the Northern Manhattan community about clinical research; enhancing recruitment from the community; and disseminating research findings to the community, including patients and families, clinicians, community organizations, community leaders, and policy makers.

\section{Reinforcing Factors}

The reinforcing factors identified in the survey and expressed in the focus group regarding clinicians' desire for improving care in their own practice are consistent with a key focus of many PBRNs. ${ }^{27} \mathrm{Re}-$ wards such as recognition as a collaborator or PBRN member and continuing medical education credit were identified as incentives by more than half the survey sample and are relatively easy and low in cost to implement.

\section{Limitations}

The primary limitations of our study relate to the size of the sample and the bias inherent in a volunteer sample of clinicians choosing to participate in a survey, focus group, or interview related to clinical research. In addition, independent Hispanic physicians were purposively chosen based on their research experience and research interest. Consequently, we are uncertain about the generalizability of our study. Moreover, given the relatively small number of focus groups and interviews, it is possible that additional themes might have emerged if we had continued to collect data.

\section{Conclusion}

Our study findings suggest that it is feasible to implement clinical research in the urban community medical practices that we studied. The ACN clinicians who participated in the survey and focus group displayed a strong interest in clinical research, and the community-based Hispanic physicians and DNPs expressed sufficient research interest to warrant further exploration of their practice sites as future PBRN members. Enabling factors are a key area on which we need to focus efforts to increase the research productivity and efficiency of the existing PBRN and to recruit future sites. Concurrently, we need to create incentives that are attractive to current and future PBRN members. The lessons that we have learned may be helpful to others in similar settings. 
The authors thank Martha Rodriguez for her assistance in facilitating the qualitative data collection and Dr. Chunhua Weng for her assistance in implementing the survey.

\section{References}

1. Sung NS, Crowley WF Jr, Genel M, et al. Central challenges facing the national clinical research enterprise. JAMA 2003;289:1278-87.

2. Zerhouni E. The NIH Roadmap. Science 2003;302: 63-72.

3. Zerhouni EA. US biomedical research: basic, translational, and clinical sciences. JAMA 2005;294:1352-8.

4. Zerhouni EA. Translational and clinical sciencetime for a new vision. N Engl J Med 2005;353: 1621-3.

5. National Institutes of Health. NIH Roadmap for medical research. Re-engineering the Clinical Research Enterprise. Available at: http://nihroadmap. nih.gov/clinicalresearchtheme/. Accessed February 13, 2008.

6. National Institutes of Health. Part I overview information. Limited competition for supplements to CTSAs to plan for pilot projects to apply the National Clinical Research Associates Model in their community engagement activities (UL1). Available at: http:/grants.nih.gov/grants/guide/rfa-files/RFARM-07-006.html. Accessed February 13, 2008.

7. Graham DG, Spano MS, Stewart TV, Staton EW, Meers A, Pace WD. Strategies for planning and launching PBRN research studies: a project of the Academy of Family Physicians National Research Network (AAFP NRN). J Am Board Fam Med 2007; 20:220-8.

8. Tierney WM, Oppenheimer CC, Hudson BL, et al. A national survey of primary care practice-based research networks. Ann Fam Med 2007;5:242-50.

9. Agency for Healthcare Research and Quality. PBRN networks. Available at: http://pbrn.ahrq.gov/portal/ server.pt ?open $=512 \&$ obj $I D=855 \&$ mode $=2 \&$ cached $=$ true\#search. Accessed January 21, 2009.

10. Conway PH, Clancy C. Transformation of health care at the front line. JAMA 2009;301:763-5.

11. Peterson KA. National Institutes of Health eliminates funding for national architecture linking primary care research. J Am Board Fam Med 2007;20: 229-31.

12. Green LW, Kreuter MW. Health promotion planning: an educational and ecological approach. 4th ed. Boston, MA: McGraw Hill; 2005.

13. Green $L W$. What can we generalize from research on patient education and clinical health promotion to physician counseling on diet? Eur J Clin Nutr 1999;53(Suppl 2):S9-S18.
14. Mundinger MO. Who's who in nursing: bringing clarity to the doctor of nursing practice. Nurs Outlook 2005;53:173-6.

15. Ramirez AG, Wildes K, Talavera G, NapolesSpringer A, Gallion K, Perez-Stable EJ. Clinical trials attitudes and practices of Latino physicians. Contemp Clin Trials 2008;29:482-92.

16. Collaborative Institutional Training Initiative. CITI login and registration. Available at: https://www. citiprogram.org/default.asp?language $=$ english. Accessed January 24, 2009.

17. The Academy of Pharmaceutical Physicians and Investigators. [Homepage] Available at: http://www. appinet.org/default.aspx. Accessed January 24, 2009.

18. Association of Clinical Research Professionals. Classroom learning. Available at: http://www. acrpnet.org/MainMenuCategory/Education/ClassroomLearning.aspx. Accessed January 24, 2009.

19. Association of Clinical Research Professionals. Investigator curriculum. Available at: http://www. acrpnet.org/MainMenuCategory/Education/online/ OnlineClinicalCourses/InvestigatorCurriculum.aspx. Accessed January 24, 2009.

20. American Academy of Family Physicians. Policies of the National Research Network. Available at: http:// www.aafp.org/online/en/home/clinical/research/ natnet/nrnpolicies.html. Accessed January 24, 2009.

21. Bigger JT, Busacca L, Florenz M, Slavik W, Steinman R, Johnson S. Re-engineering clinical research: a scalable model integrating financial management with workflow in community clinical research networks. Bethesda, MD: National Leadership Forum; 2006.

22. Kho A, Zafar A, Tierney W. Information technology in PBRNs: the Indiana University Medical Group Research Network (IUMG ResNet) experience. J Am Board Fam Med 2007;20:196-203.

23. Practice Partner Research Network. [Homepage] Available at: http://www.musc.edu/PPRNet/. Accessed January 24, 2009.

24. Ford JG, Howerton MW, Lai GY, et al. Barriers to recruiting underrepresented populations to cancer clinical trials: a systematic review. Cancer 2008;112: 228-42.

25. Napoles-Springer A, Grumbach K, Alexander M, et al. Clinical research with older African Americans and Latinos. Res Aging 2000;22:668-91.

26. Calderon JL, Baker RS, Fabrega H, et al. An ethnomedical perspective on research participation: a qualitative pilot study. MedGenMed 2006;8:23.

27. Mold JW, Peterson KA. Primary care practice-based research networks: working at the interface between research and quality improvement. Ann Fam Med 2005;3(Suppl 1):S12-20. 